\title{
Diagnostic value of the lymphocyte-to-monocyte and albumin-to-globulin ratios in patients with colon cancer
}

\section{Li Huang}

Guangxi Medical University First Affiliated Hospital

\section{Zhuning Mo}

People's Hospital of Guangxi Zhuang Autonomous Region

\section{Zuojian Hu}

Guangxi Medical University First Affiliated Hospital

\section{Linyan Zhang}

Guangxi Medical University First Affiliated Hospital

\section{Shanzi Qin}

Guangxi Medical University First Affiliated Hospital

\section{Xue Qin}

Guangxi Medical University First Affiliated Hospital

Shan Li ( $\sim$ lis8858@126.com )

Guangxi Medical University First Affiliated Hospital

\section{Research article}

Keywords: lymphocyte-to-monocyte ratio, albumin-to-globulin ratio, colon cancer, diagnosis

Posted Date: March 30th, 2020

DOl: https://doi.org/10.21203/rs.3.rs-18853/v1

License: (c) (i) This work is licensed under a Creative Commons Attribution 4.0 International License. Read Full License 


\section{Abstract}

Background: Ample evidence has revealed that the lymphocyte-to-monocyte ratio (LMR) and albumin-toglobulin ratio (AGR) are cancer-related inflammatory markers. The present study aimed to assess whether LMR and AGR, alone or in combination with carcinoembryonic antigen (CEA), was a useful diagnostic marker for colon cancer.

Methods: This retrospective study enrolled 251 patients with colon cancer, 171 patients with benign colon diseases, and 187 healthy control subjects from January 2012 to September 2019. Mann-Whitney U test or Chi-square test were used to analyze differences between groups in laboratory parameters and clinicopathological features. The diagnostic value of LMR and AGR combined or not with CEA in colon cancer patients was determined via a receiver operating characteristic (ROC) curve.

Results: The levels of LMR and AGR were decreased in the colon cancer group compared with the healthy control and benign colon disease groups. The LMR and AGR were correlated with lymph node metastasis, tumor size, and clinical stage. Moreover, AGR was associated with distant metastasis. Both the LMR ( $r=$ -0.137, $p=0.030)$ and AGR $(r=-0.178, p=0.005)$ were negatively correlated with the concentration of CEA. LMR or AGR combined with CEA could enhance sensitivity $(75.30 \%$ for LMR + CEA, $58.57 \%$ for AGR + CEA) and generate larger area under curve (AUC; 0.75 for LMR + CEA, 0.74 for AGR + CEA) compared with the LMR $(p<0.001)$, AGR $(p<0.001)$, or CEA $(p<0.001)$ alone.

Conclusion: The combination of LMR or AGR with CEA may enhance the sensitivity and diagnostic efficacy of detecting colon cancer from benign colon diseases.

\section{Background}

Colon cancer comprised the highest incidence in tumors of the digestive system and represented a commonly diagnosed malignant tumor worldwide in 2018[1]; it was the fourth most frequent cause of cancer morbidity and fifth leading cause of cancer mortality in China in 2015[2]. Due to the lack of obvious clinical manifestations, most colon cancer patients have silent symptoms for years. More than $50 \%$ of colon cancer patients are clinically diagnosed at the advanced cancer stage[3], and the 5-year survival rate is only $10 \%[4]$. However, with the improvements of modern medicine and surgical techniques, the 5-year survival rate for early stage colon cancer patients reaches up to $90 \%$ [4]. Hence, early screening and diagnosis is an effective method for the prevention and treatment of colon cancer that will improve clinical outcome. Fecal occult blood test (FOBT) is a cheap and convenient screening method for colon cancer, while the results are affected by diet, drugs, and bleeding[5]. Colonoscopy and biopsy have been used as ideal methods for the diagnosis of early colon cancer, but these tests greatly increase the physical and financial burden on patients harboring colonic diseases, resulting in poor compliance. Hence, low-cost and easily obtainable markers have important significance for the early diagnosis and prevention of colon cancer. 
Several serum tumor markers have been commonly used in colon diseases, particularly carcinoembryonic antigen (CEA). Previous studies revealed that CEA possessed low sensitivity and could not appropriately present the complete potency for predicting and monitoring the clinical diagnosis and treatment in colorectal cancer[6]. In recent years, many inflammatory parameters from peripheral blood and serum have been evaluated in the diagnosis and prognosis of multiple malignancies, including colorectal cancer[7, 8], such as circulating neutrophils[9], lymphocytes[10], monocytes[11], platelets[12], and albumin[13]. As crucial components of host immunity, lymphocytes can infiltrate into the tumor microenvironment, and they play a vital role in cell-mediated immunity, preventing the proliferation and metastatic activity of colorectal cancer[14]. Systemic inflammation can induce changes in the hematological system, leading to a significant decrease of lymphocytes[15, 16]. Lymphocytopenia is considered an insufficient immune response against tumor, resulting in hyperproliferation and tumorigenesis. Conversely, the excess circulating monocytes gather and settle in solid tumor tissues after being mediated by chemokines of inflammatory cytokines; they are then differentiated into tumorassociated macrophages with specific phenotypic characteristics[17]. Increasing evidence has demonstrated that the accumulation of tumor-associated macrophages in the tumor sites contributes to the angiogenesis, tumorigenesis, and pathogenesis of colon cancer[18]. As a result, the relatively lower number of lymphocytes is an indicator marker of weak immune response, and the elevated monocyte count is a microenvironment monitor of high tumor burden. Therefore, lymphocyte to monocyte ratio $(\mathrm{LMR})$, as a reflection of systemic inflammation and immunological statuses, may have a crucial role in the progression of colon cancer.

The albumin-to-globulin ratio (AGR) which combines serum albumin and globulin, is a routinely available and cost-effective marker and associated with the process of inflammatory and nourishment state. Accumulated evidence displayed that AGR was an independent and useful predictor in the prognosis of colon cancer by regulating cells and/or releasing several mediators[19]; moreover, elevated AGR was a favorable factor for better clinical outcomes[8]. Hence, we hypothesized that AGR may have a significant diagnostic value in colon cancer.

Up to now, studies have rarely investigated the diagnostic role of these inflammatory parameters (LMR and AGR) in colon cancer. Therefore, this study examined the value of LMR and AGR combined or not with CEA in distinguishing colon cancer from benign colon diseases.

\section{Methods}

\section{Patients}

For the present study, 251 colon cancer patients, 171 patients with benign colon diseases, and 187 healthy controls were recruited at the First Affiliated Hospital of Guangxi Medical University in January 2012 to September 2019. In colon cancer participants with new diagnoses, the disease was confirmed by histology and treated with surgical resection. Clinical staging of colon cancer was conducted accorded to the seventh edition of the American Joint Committee on Cancer/TNM tumor staging criteria. Patients with 
other cancers, cardiovascular disease, diabetes mellitus, hematological disease, autoimmune disease, recent blood transfusion, or treatment with other therapies, such as radiotherapy and chemotherapy, were excluded. Colon polyps, colon adenomas, and colonitis were included as benign colon disease patients who were diagnosed by colonoscopy and histopathology. Healthy controls comprised healthy subjects undergoing physical examination during the same period. This research was approved by the Ethics Committee of the First Affiliated Hospital of Guangxi Medical University.

\section{Methods}

All data were collected from the hospital's electronic medical records, including gender, age, white blood cells (WBC), platelets, hemoglobin, lymphocyte, monocyte, albumin, globulin, and CEA. Whole blood-cell parameters were detected with a Beckmann 780 device (Beckman Coulter, Brea, CA). The levels of albumin and total protein were analyzed by a Hitachi 7600 automatic biochemical analyzer (Hitachi HighTechnologies Corporation, Tokyo, Japan). The concentration of serum CEA was tested by using a Roche E6000 analyzer (Roche Diagnostics, Basel, Switzerland). The ratios of interest were calculated as follows: LMR = lymphocyte count / monocyte count and AGR = albumin / (total protein - albumin).

\section{Statistical analysis}

The Kolmogorov-Smirnov test was used to detect the distribution of the continuous variables. All the data failed to satisfy the normal distribution; median and interquartile ranges were applied for nonnormal data. Differences between groups in laboratory parameters and clinical characteristics were calculated using the Mann-Whitney nonparametric $U$ test or Chi-square test. The Spearman correlation coefficient was conducted to detect correlations between LMR or AGR and CEA in the colon cancer group. The receiver operating characteristic (ROC) curve and area under the curve (AUC) were determined by MedCalc statistical software (version 18.1.1). The SPSS 16.0 statistical software package was used for data processing and analysis, using a significance level of 0.05 .

\section{Results}

\section{Patient characteristics}

Basic information and laboratory parameters are summarized in Table 1. The median age of the colon cancer patients, benign colon diseases cases, and control individuals were 56 (46-64), 48 (41-56), and 53 (49-60) years, respectively. No intergroup difference was observed in gender among the three groups. The measured results for WBCs, platelets, monocytes, and CEA were higher in the colon cancer patients compared with those in the healthy and benign colon disease subjects. Conversely, the levels of median hemoglobin, lymphocytes, albumin, LMR, and AGR were significantly lower in the colon cancer group than they were in the control and benign colon disease groups, and there were statistical differences in LMR (Fig. 1A) and AGR (Fig. 1B) among the three groups. 


\section{Correlations between LMR, AGR, and CEA and the clinicopathological characteristics in patients with colon cancer}

There was a negative correlation presented between CEA and the LMR $(r=-0.137, p=0.030$, Fig. 2A) and $\operatorname{AGR}(r=-0.178, p=0.005$, Fig. 2B) in the colon cancer group, respectively. According to the seventh edition of the American Joint Committee on Cancer/TNM tumor stage, the clinicopathological characteristics of the 251 patients carrying colon cancer are shown in Table 2. The levels of LMR and AGR in the colon cancer group were both closely related to the clinical features, including lymph node metastasis, tumor size, and clinical stage, but they were not associated with serosa invasion. Moreover, the AGR and CEA were correlated with distant metastasis.

\section{Diagnostic efficacy of LMR, AGR, and CEA alone or in combination to differentiate colon cancer from benign colon tumors}

The results of the ROC curve analysis are presented in Table 3 and Fig. 3. To predict colon cancer, the optimal cutoffs of the LMR, AGR, CEA were 4.58 (AUC $=0.69$, sensitivity $=77.29 \%$, specificity $=53.22 \%$, positive predictive value $[\mathrm{PPV}]=70.8 \%$, and negative predictive value $[\mathrm{NPV}]=61.5 \%), 1.71(\mathrm{AUC}=0.66$, sensitivity $=83.27 \%$, specificity $=42.11 \%, \mathrm{PPV}=67.9 \%$, and NPV $=63.2 \%)$, and 3.44 (AUC $=0.69$, sensitivity $=43.43 \%$, specificity $=87.72 \%$, PPV $=83.8 \%$, and NPV $=88.8 \%$, respectively. AGR had the highest sensitivity in differentiating colon cancer from benign colon diseases, whereas CEA had the highest specificity. The AUC value indicated that CEA and LMR possessed better ability than the AGR did. The sensitivity was elevated in the combination of LMR or AGR and CEA (75.30\% for LMR + CEA; $58.57 \%$ for AGR + CEA), but the specificity decreased (63.74\% for LMR + CEA; 76.02\% for AGR + CEA). CEA combined with the LMR or AGR possessed a larger AUC value ( 0.75 for LMR + CEA; 0.74 for AGR + CEA) than the LMR $(p<0.0001)$, AGR $(p<0.0001)$ or CEA $(p<0.0001)$ alone.

\section{Discussion}

Colon cancer is closely associated with inflammation which has been unraveled as a crucial hallmark in all the steps of colon tumorigenesis, including initiation, invasion, progression, and metastasis[20]. Recent work has elucidated that cancer-associated inflammatory markers are increasingly used in the early diagnosis and prognosis of malignant tumors and closely related with the progression of diseases[21, 22]. Therefore, this study assessed the diagnostic efficacy of two common inflammatory indexes (LMR and AGR) and detected whether they could be used as surrogate markers for distinguishing colon cancer from benign colon diseases.

In this retrospective analysis, we found that the colon cancer patients had a lower LMR level than the benign colon disease patients and healthy controls did, and this was consistent with previous findings. For example, Evrim et al.[23] observed that the level of monocyte-to-lymphocyte ratio (MLR) was higher in the gastric cancer group than it was in the intestinal metaplasia and healthy control groups. Luo et al.[24] found that MLR was significantly elevated in patients carrying urothelial carcinoma of the bladder relative to healthy controls. A study by Ozawa et al.[25] demonstrated that cancer-specific survival was 
significantly worse in patients with low LMR levels than in high-LMR patients, and LMR may be an independent prognostic marker for stage III and IV colon cancer patients[26]. Moreover, our study disclosed that the level of LMR was significantly correlated with lymph node metastasis, tumor size, and clinical stage. Indeed, Peng et al.[19] assessed the prognosis of patients harboring colorectal liver-only metastases and elucidated that elevated LMR predicted a favorable outcome in both 5-year recurrencefree survival and overall survival of patients with lymph node metastases and liver tumor up to a diameter of less than $5 \mathrm{~cm}$. Furthermore, several meta-analyses have demonstrated that malignant patients with high preoperative LMR have better predicted clinical outcomes compared with patients with low LMR in populations comprising Asians, digestive system carcinomas, non-metastatic diseases and early disease stages[27, 28], which confirmed our findings.

Emerging evidence suggests that AGR is mainly used as a clinical indicator for several kinds of cancers. Growing tumors induce hypoalbuminemia via secreting inflammatory cytokines, which may inhibit albumin synthesis and promote albumin loss, resulting in weak systemic response. Rasouli et al.[29] reported that patients with malignant tumors had a decreased concentration of albumin, which were measured by colorimetric methods, compared with healthy controls, which was accordant with the present study results. Globulin, as a reflector for most proinflammation protein, was increased by the accumulation of acute-phase proteins and immunoglobulins[30]. The AGR, which is compatible with hypoalbuminemia and hyperglobulinemia, may be able to more accurately reflect the nutritional and inflammatory state, and thus, is associated with the progression of neoplasia. The electrophoretic data of serum proteins showed that the AGR was significantly decreased in 85 patients harboring cancer relative to controls[29]. Cheng et al.[31] confirmed that the globulin-to-albumin ratio (GAR), was significantly higher in the subjects with liver disease compared with individuals with no evidence of liver disease. Quite a few studies revealed that patients with lower pretreatment AGR were related to worse survival than higher AGR subjects in colorectal cancer[32], gastric cancer[33], pancreatic cancer[34], nasopharyngeal carcinoma[35], and esophageal cancer[36]. Moreover, a significant correlation based on the abovementioned researches was observed between clinical characteristics and the level of AGR, such as lymph node metastasis, tumor size, distant metastasis, and tumor stage. In agreement with previous studies, this study found that the value of AGR in the colon cancer patients was lower than that in the benign colon diseases and healthy individuals; furthermore, it showed that the AGR was associated with lymph node metastasis, distant metastasis, tumor size, and clinical stage.

CEA is a serum glycoprotein that is mainly secreted by cells of the large intestine, and it has been widely applied as a tumor marker for the malignant characteristics of colorectal cancer. Unfortunately, high levels of CEA are not present in about $15 \%$ of large intestine cancers, and elevated CEA is commonly revealed in severe malignant tumors[37]. In clinical practice, increased circulating levels of CEA are observed not only in cancer patients but also in some benign intestinal lesions. Therefore, the sensitivity and effectiveness of CEA are not sufficient for clinical diagnosis and treatment, but CEA has a high specificity in colorectal cancer[38,39], as well as in differentiating colorectal cancer patients from those with benign colorectal diseases[40]. Consistent with previous studies, the sensitivity and diagnostic value of CEA were not noticeable in identifying colon cancer from benign colon diseases $(43.43 \%, 0.69)$, while 
the specificity was up to $87.72 \%$. In addition, we found that CEA combined with LMR or AGR generated a significantly higher diagnostic sensitivity than CEA used alone did. LMR or AGR combined with CEA possessed a better AUC than LMR, AGR, or CEA used alone in discriminating colon cancer patients and benign colon diseases cases. Similar to this pilot study, a previous report displayed that LMR possessed a moderate ability $(A \cup C=0.71)$ and could contribute to distinguishing patients carrying gastric cancer from those with intestinal metaplasia[23]. In many malignancies, AGR exhibited good diagnostic efficacy (AUC $=0.81)$ in differentiating cancer patients from healthy controls[29]. All these findings suggest that the combination of CEA with LMR or AGR could not only be used as a colon cancer diagnostic biomarker but may also improve the diagnostic efficiency of detecting patients harboring colon cancer from those with benign colon diseases.

There are certain potential limitations in the current research. On the one hand, this is a retrospective analysis of a relatively small sample size from a single center, so selection bias and statistical validity should be noted, which may affect the final results about the associations between the LMR or AGR and colon cancer. We failed to stratify benign colon diseases due to the relatively small sample size. On the other, confounding factors, including dietary habits and family histories, cannot be completely ruled out, which may prevent us from drawing any firm conclusions. Therefore, a large-scale, prospective study with multiple centers is still needed to validate these results.

\section{Conclusion}

This study first described that the LMR and AGR were correlated with CEA and colon cancer, as well as lymph node metastasis, tumor size, and clinical stage. Moreover, it illustrated that AGR was associated with distant metastasis. The combination of the LMR or AGR with CEA can enhance sensitivity and diagnostic efficacy and may be a helpful diagnostic marker for differentiating colon cancer from benign colon diseases.

\section{Abbreviations}

WBC: white blood cells; ROC: receiver-operating characteristic curve; AUC: area under curve; Cl: confidence interval; FOBT: fecal occult blood test; LMR: lymphocyte-to-monocyte ratio; AGR: albumin-to-globulin ratio; CEA: carcinoembryonic antigen; PLR: positive likelihood ratio; NLR: negative likelihood ratio; PPV: positive predictive value; NPV: negative predictive value; GAR: globulin-to-albumin ratio; MLR: monocyteto-lymphocyte ratio

\section{Declarations}

\section{Ethical Approval and consent to participate}

The present study was approved by the Ethics Committee of the First Affiliated Hospital of Guangxi Medical University, and informed consent was obtained from all the participants. 


\section{Author's contributions}

SL drafted the overall design of this paper as the corresponding authors. LH, ZM

and ZH wrote the article. LZ collected the laboratory data. SQ and XQ analyzed the data.

\section{Funding}

This work was supported by the Youth Science Foundation of The People's Hospital of Guangxi Zhuang Autonomous Region『QN2018-7区.

\section{Availability of data and materials}

The datasets used and/or analyzed during the current study are available

from the corresponding author on reasonable request.

\section{Consent for publication}

Not applicable.

\section{Competing interests}

The authors declare that they have no competing interests.

\section{Acknowledgments}

We thank Scribendi.com for its linguistic assistance during the preparation of this manuscript.

\section{Authors' information}

${ }^{1}$ Department of Clinical Laboratory, First Affiliated Hospital of Guangxi Medical University, Nanning, Guangxi Zhuang Autonomous Region, China.

${ }^{2}$ Department of Blood Transfusion, The People's Hospital of Guangxi Zhuang Autonomous Region, Nanning, Guangxi Zhuang Autonomous Region, China.

\section{References}

1. Bray, F., J. Ferlay, I. Soerjomataram, R.L. Siegel, L.A. Torre, and A. Jemal, Global cancer statistics 2018: GLOBOCAN estimates of incidence and mortality worldwide for 36 cancers in 185 countries. CA Cancer J Clin, 2018;68(6):394-424.

2. Chen, W., C. Xia, R. Zheng, M. Zhou, C. Lin, H. Zeng, S. Zhang, L. Wang, Z. Yang, K. Sun, et al., Disparities by province, age, and sex in site-specific cancer burden attributable to 23 potentially 
modifiable risk factors in China: a comparative risk assessment. Lancet Glob Health, 2019;7(2):e257e269.

3. Siegel, R.L., K.D. Miller, S.A. Fedewa, D.J. Ahnen, R.G.S. Meester, A. Barzi, and A. Jemal, Colorectal cancer statistics, 2017. CA Cancer J Clin, 2017;67(3):177-193.

4. Brenner, H., M. Kloor, and C.P. Pox, Colorectal cancer. Lancet, 2014;383(9927):1490-1502.

5. Lee, C.S., L. Ronan, C. O'Morain, and D. McNamara, Screening for colorectal cancer: what fits best? Expert Rev Gastroenterol Hepatol, 2012;6(3):301-12.

6. Yamashita, K. and M. Watanabe, Clinical significance of tumor markers and an emerging perspective on colorectal cancer. Cancer Sci, 2009;100(2):195-9.

7. Tan, D., Y. Fu, W. Tong, and F. Li, Prognostic significance of lymphocyte to monocyte ratio in colorectal cancer: A meta-analysis. Int J Surg, 2018;55:128-138.

8. Fujikawa, H., Y. Toiyama, Y. Inoue, H. Imaoka, T. Shimura, M. Okigami, H. Yasuda, J. Hiro, S. Yoshiyama, S. Saigusa, et al., Prognostic Impact of Preoperative Albumin-to-Globulin Ratio in Patients with Colon Cancer Undergoing Surgery with Curative Intent. Anticancer Res, 2017;37(3):1335-1342.

9. Shaul, M.E. and Z.G. Fridlender, Cancer-related circulating and tumor-associated neutrophils subtypes, sources and function. FEBS J, 2018;285(23):4316-4342.

10. Iseki, Y., M. Shibutani, K. Maeda, H. Nagahara, T. Tamura, G. Ohira, S. Yamazoe, K. Kimura, T. Toyokawa, R. Amano, et al., The impact of the preoperative peripheral lymphocyte count and lymphocyte percentage in patients with colorectal cancer. Surg Today, 2017;47(6):743-754.

11. Valero, C., D.K. Zanoni, M.R. McGill, I. Ganly, L.G.T. Morris, M. Quer, J.P. Shah, R.J. Wong, X. Leon, and S.G. Patel, Pretreatment peripheral blood leukocytes are independent predictors of survival in oral cavity cancer. Cancer, 2019,https://doi.org/. 10.1002/cncr.32591.

12. Rossaint, J., A. Margraf, and A. Zarbock, Role of Platelets in Leukocyte Recruitment and Resolution of Inflammation. Front Immunol, 2018;9:2712.

13. Kashiwada, T., Y. Saito, Y. Terasaki, K. Hisakane, S. Takeuchi, T. Sugano, A. Miyanaga, R. Noro, Y. Minegishi, M. Seike, et al., Interstitial lung disease associated with nanoparticle albumin-bound paclitaxel treatment in patients with lung cancer. Jpn J Clin Oncol, 2019;49(2):165-173.

14. Bindea, G., B. Mlecnik, M. Tosolini, A. Kirilovsky, M. Waldner, A.C. Obenauf, H. Angell, T. Fredriksen, L. Lafontaine, A. Berger, et al., Spatiotemporal dynamics of intratumoral immune cells reveal the immune landscape in human cancer. Immunity, 2013;39(4):782-95.

15. Lin, W.W. and M. Karin, A cytokine-mediated link between innate immunity, inflammation, and cancer. J Clin Invest, 2007;117(5):1175-83.

16. Menges, T., J. Engel, I. Welters, R.M. Wagner, S. Little, R. Ruwoldt, M. Wollbrueck, and G. Hempelmann, Changes in blood lymphocyte populations after multiple trauma: association with posttraumatic complications. Crit Care Med, 1999;27(4):733-40. 
17. De la Fuente Lopez, M., G. Landskron, D. Parada, K. Dubois-Camacho, D. Simian, M. Martinez, D. Romero, J.C. Roa, I. Chahuan, R. Gutierrez, et al., The relationship between chemokines CCL2, CCL3, and CCL 4 with the tumor microenvironment and tumor-associated macrophage markers in colorectal cancer. Tumour Biol, 2018;40(11):1010428318810059.

18. Kvorjak, M., Y. Ahmed, M.L. Miller, R. Sriram, C. Coronnello, J.G. Hashash, D.J. Hartman, C.A. Telmer, N. Miskov-Zivanov, O.J. Finn, et al., Crosstalk between colon cells and macrophages increases ST6GALNAC1 and MUC1-sTn expression in ulcerative colitis and colitis-associated colon cancer. Cancer Immunol Res, 2019,https://doi.org/. 10.1158/2326-6066.CIR-19-0514.

19. Peng, J., H. Li, Q. Ou, J. Lin, X. Wu, Z. Lu, Y. Yuan, D. Wan, Y. Fang, and Z. Pan, Preoperative lymphocyte-to-monocyte ratio represents a superior predictor compared with neutrophil-tolymphocyte and platelet-to-lymphocyte ratios for colorectal liver-only metastases survival. Onco Targets Ther, 2017;10:3789-3799.

20. Terzic, J., S. Grivennikov, E. Karin, and M. Karin, Inflammation and colon cancer. Gastroenterology, 2010;138(6):2101-2114 e5.

21. Li, Z., Z. Xu, Y. Huang, R. Zhao, Y. Cui, Y. Zhou, and X. Wu, Prognostic values of preoperative plateletto-lymphocyte ratio, albumin and hemoglobin in patients with non-metastatic colon cancer. Cancer Manag Res, 2019;11:3265-3274.

22. Zhang, H., B. Zhang, K. Zhu, C. Wu, L. Gao, X. Sun, C. Liu, and C. Wang, Preoperative albumin-toglobulin ratio predicts survival in patients with non-small-cell lung cancer after surgery. J Cell Physiol, 2019;234(3):2471-2479.

23. Aksoy, E.K., S. Kantarci, M. Torgutalp, M.Y. Akpinar, F.P. Sapmaz, G.S. Yalcin, M. Uzman, G.G. Simsek, and Y. Nazligul, The importance of complete blood count parameters in the screening of gastric cancer. Prz Gastroenterol, 2019;14(3):183-187.

24. Luo, Y., X. Shi, W. Li, L. Mo, Z. Yang, X. Li, L. Qin, and W. Mo, Evaluation of the clinical value of hematological parameters in patients with urothelial carcinoma of the bladder. Medicine (Baltimore), 2018;97(14):e0351.

25. Ozawa, T., S. Ishihara, K. Kawai, S. Kazama, H. Yamaguchi, E. Sunami, J. Kitayama, and T. Watanabe, Impact of a lymphocyte to monocyte ratio in stage IV colorectal cancer. J Surg Res, 2015;199(2):38692.

26. Stotz, M., M. Pichler, G. Absenger, J. Szkandera, F. Arminger, R. Schaberl-Moser, H. Samonigg, T. Stojakovic, and A. Gerger, The preoperative lymphocyte to monocyte ratio predicts clinical outcome in patients with stage III colon cancer. Br J Cancer, 2014;110(2):435-40.

27. Nishijima, T.F., H.B. Muss, S.S. Shachar, K. Tamura, and Y. Takamatsu, Prognostic value of lymphocyte-to-monocyte ratio in patients with solid tumors: A systematic review and meta-analysis. Cancer Treat Rev, 2015;41(10):971-8.

28. Teng, J.J., J. Zhang, T.Y. Zhang, S. Zhang, and B.S. Li, Prognostic value of peripheral blood lymphocyte-to-monocyte ratio in patients with solid tumors: a meta-analysis. Onco Targets Ther, 2016;9:37-47. 
29. Rasouli, M., A. Okhovatian, and A. Enderami, Serum proteins profile as an indicator of malignancy: multivariate logistic regression and ROC analyses. Clin Chem Lab Med, 2005;43(9):913-8.

30. Gabay, C. and I. Kushner, Acute-phase proteins and other systemic responses to inflammation. N Engl J Med, 1999;340(6):448-54.

31. Cheng, K.C., W.Y. Lin, C.S. Liu, C.C. Lin, H.C. Lai, and S.W. Lai, Association of different types of liver disease with demographic and clinical factors. Biomedicine (Taipei), 2016;6(3):16.

32. Hachiya, H., M. Ishizuka, K. Takagi, Y. Iwasaki, N. Shibuya, Y. Nishi, T. Aoki, and K. Kubota, Clinical significance of the globulin-to-albumin ratio for prediction of postoperative survival in patients with colorectal cancer. Ann Gastroenterol Surg, 2018;2(6):434-441.

33. Xiao, S., F. Feng, N. Liu, Z. Liu, Y. Guo, X. Lian, and H. Zhang, Preoperative Albumin Level Is Superior To Albumin-Globulin Ratio As A Predicting Indicator In Gastric Cancer Patients Who Underwent Curative Resection. Cancer Manag Res, 2019;11:9931-9938.

34. Feng, L., S. Gu, P. Wang, H. Chen, Z. Chen, Z. Meng, and L. Liu, Pretreatment values of bilirubin and albumin are not prognostic predictors in patients with advanced pancreatic cancer. Cancer Med, 2018;7(12):5943-5951.

35. Li, A.C., W.W. Xiao, L. Wang, G.Z. Shen, A.A. Xu, Y.Q. Cao, S.M. Huang, C.G. Lin, F. Han, X.W. Deng, et al., Risk factors and prediction-score model for distant metastasis in nasopharyngeal carcinoma treated with intensity-modulated radiotherapy. Tumour Biol, 2015;36(11):8349-57.

36. Oki, S., Y. Toiyama, Y. Okugawa, T. Shimura, M. Okigami, H. Yasuda, H. Fujikawa, Y. Okita, S. Yoshiyama, J. Hiro, et al., Clinical burden of preoperative albumin-globulin ratio in esophageal cancer patients. Am J Surg, 2017;214(5):891-898.

37. Lawicki, S., B. Mroczko, and M. Szmitkowski, [Neoplasm markers useful for diagnosis and monitoring of colonic neoplasms]. Postepy Hig Med Dosw, 2002;56(5):617-34.

38. Theron, E.J., C.F. Albrecht, P.B. Kruger, K. Jenkins, and M.J. van der Merwe, beta-Glucosidase activity in fetal bovine serum renders the plant glucoside, hypoxoside, cytotoxic toward B16-F10-BL-6 mouse melanoma cells. In Vitro Cell Dev Biol Anim, 1994;30A(2):115-9.

39. Locker, G.Y., S. Hamilton, J. Harris, J.M. Jessup, N. Kemeny, J.S. Macdonald, M.R. Somerfield, D.F. Hayes, R.C. Bast, Jr., and Asco, ASCO 2006 update of recommendations for the use of tumor markers in gastrointestinal cancer. J Clin Oncol, 2006;24(33):5313-27.

40. Holdenrieder, S., P. Stieber, V. Liska, V. Treska, O. Topolcan, J. Dreslerova, V.M. Matejka, J. Finek, and L. Holubec, Cytokeratin serum biomarkers in patients with colorectal cancer. Anticancer Res, 2012;32(5):1971-6.

\section{Tables}

Table 1. Basic information and laboratory parameters among colon cancer, benign colon diseases and healthy control groups. 


\begin{tabular}{lllllll}
\hline is & $\begin{array}{l}\text { Healthy controls } \\
(\mathrm{N}=187)\end{array}$ & Benign colon diseases $(\mathrm{N}=171)$ & Colon cancer $(\mathrm{N}=251)$ & $P^{a}$ & $P^{b}$ & $P^{C}$ \\
& & & & & & \\
\hline /Female) & $99 / 88$ & $90 / 81$ & $140 / 111$ & 0.557 & 0.525 & 0.953 \\
& $53.0(49.0-60.0)$ & $48.0(41.0-56.0)$ & $56.0(46.0-64.0)$ & 0.092 & $<0.001$ & $<0.001$ \\
& $6.10(5.17-6.71)$ & $6.30(5.20-7.46)$ & $6.41(5.33-7.70)$ & $<0.001$ & 0.453 & 0.028 \\
//L) & $143.00(135.10-150.60)$ & $131.00(120.00-142.70)$ & $117.00(99.00-130.80)$ & $<0.001$ & $<0.001$ & $<0.001$ \\
$/ \mathrm{L})$ & $202.20(179.50-227.80)$ & $230.20(193.60-276.50)$ & $275.00(228.50-345.00)$ & $<0.001$ & $<0.001$ & $<0.001$ \\
$\left.\times 10^{9} / \mathrm{L}\right)$ & $2.03(1.74-2.41)$ & $2.10(1.64-2.63)$ & $1.83(1.55-2.22)$ & $<0.001$ & $<0.001$ & 0.381 \\
$\left.0^{9} / \mathrm{L}\right)$ & $0.41(0.33-0.50)$ & $0.48(0.37-0.58)$ & $0.50(0.41-0.62)$ & $<0.001$ & 0.012 & $<0.001$ \\
& $46.70(45.0-48.20)$ & $42.60(39.40-45.20)$ & $38.50(36.10-41.10)$ & $<0.001$ & $<0.001$ & $<0.001$ \\
& $27.10(25.30-29.20)$ & $26.00(23.20-28.70)$ & $26.10(23.70-28.80)$ & 0.001 & 0.381 & 0.000 \\
& $0.71(0.39-1.30)$ & $1.74(1.08-2.61)$ & $2.71(1.56-7.28)$ & $<0.001$ & $<0.001$ & $<0.001$ \\
& $5.13(4.24-5.87)$ & $4.67(3.67-5.60)$ & $3.63(2.86-4.51)$ & $<0.001$ & $<0.001$ & 0.002 \\
& $1.72(1.57-1.86)$ & $1.63(1.43-1.91)$ & $1.49(1.31-1.65)$ & $<0.001$ & $<0.001$ & 0.028 \\
\hline
\end{tabular}

WBC, white blood cells; CEA, carcinoembryonic antigen; LMR, lymphocyte-to-monocyte ratio; AGR, albumin-to-globulin ratio. $P^{\mathrm{a}}$ : colon cancer vs healthy controls; $P^{\mathrm{b}}$ : colon cancer vs benign colon diseases; $P^{\mathrm{c}}$ : benign colon diseases vs healthy controls.

Table 2. Correlation between LMR, AGR, and CEA and clinicopathological features in colon cancer

\begin{tabular}{|c|c|c|c|c|c|c|c|}
\hline & $\mathrm{N}$ & LMR & $P$ & AGR & $P$ & CEA & $P$ \\
\hline \multicolumn{8}{|c|}{ Tumor invasion (T stage) } \\
\hline $\mathrm{T} 1+\mathrm{T} 2$ & 86 & $3.63(2.83-4.87)$ & \multirow[t]{2}{*}{0.513} & $1.46(1.33-1.62)$ & \multirow[t]{2}{*}{0.602} & $2.50(1.51-4.54)$ & \multirow[t]{2}{*}{0.187} \\
\hline $\mathrm{T} 3+\mathrm{T} 4$ & 165 & $3.63(2.89-4.36)$ & & $1.50(1.31-1.66)$ & & $3.03(1.60-8.01)$ & \\
\hline \multicolumn{8}{|c|}{ Lymph node metastasis ( $\mathrm{N}$ stage) } \\
\hline No & 177 & $3.75(2.85-4.87)$ & \multirow[t]{2}{*}{0.033} & $1.52(1.33-1.68)$ & \multirow[t]{2}{*}{0.025} & $2.58(1.51-4.88)$ & \multirow[t]{2}{*}{0.009} \\
\hline N1-N3 & 74 & $3.44(2.90-4.12)$ & & $1.43(1.31-1.58)$ & & $4.21(1.88-12.83)$ & \\
\hline \multicolumn{8}{|c|}{ Distant metastasis (M stage) } \\
\hline M0 & 242 & $3.63(2.94-4.52)$ & \multirow[t]{2}{*}{0.366} & $1.50(1.32-1.65)$ & \multirow[t]{2}{*}{0.013} & $2.65(1.52-6.64)$ & \multirow[t]{2}{*}{0.003} \\
\hline M1 & 9 & $3.09(2.48-4.28)$ & & $1.19(1.04-1.44)$ & & $41.09(2.94-105.71)$ & \\
\hline \multicolumn{8}{|c|}{ Tumor size (cm) } \\
\hline$<5$ & 159 & $3.85(3.00-4.65)$ & \multirow[t]{2}{*}{0.004} & $1.52(1.34-1.67)$ & \multirow[t]{2}{*}{0.002} & $2.54(1.56-6.35)$ & \multirow[t]{2}{*}{0.181} \\
\hline$\geq 5$ & 92 & $3.26(2.70-4.17)$ & & $1.43(1.15-1.60)$ & & $3.54(1.50-8.56)$ & \\
\hline \multicolumn{8}{|c|}{ Clinical stage } \\
\hline $\mathrm{I}+\mathrm{II}$ & 179 & 3.77(2.95-4.86ロ & \multirow[t]{2}{*}{0.013} & $1.52(1.33-1.69)$ & \multirow[t]{2}{*}{0.002} & $2.55(1.39-5.02)$ & \multirow[t]{2}{*}{0.003} \\
\hline III + IV & 72 & $3.43(2.80-4.03)$ & & $1.44(1.23-1.57)$ & & $3.96(1.92-16.00)$ & \\
\hline
\end{tabular}

CEA, carcinoembryonic antigen; LMR, lymphocyte-to-monocyte ratio; AGR, albumin-to-globulin ratio.

Table 3. Diagnostic efficacy of LMR, AGR and CEA used alone or in combination to differentiate colon cancer frombenign colon diseases.

\begin{tabular}{lllllllll}
\hline Cutoff & Sensitivity(\%) & Specificity(\%) & PLR & NLR & PPV (\%) & NPV (\%) & AUC (95\% CI) & $P$ \\
\hline 4.58 & 77.29 & 53.22 & 1.65 & 0.43 & 70.8 & 61.5 & $0.69(0.64-0.73)$ & $<0.001$ \\
1.71 & 83.27 & 42.11 & 1.44 & 0.40 & 67.9 & 63.2 & $0.66(0.61-0.70)$ & $<0.001$ \\
3.44 & 43.43 & 87.72 & 3.54 & 0.64 & 83.8 & 88.8 & $0.69(0.64-0.73)$ & $<0.001$ \\
0.54 & 75.30 & 63.74 & 2.08 & 0.39 & 75.3 & 63.7 & $0.75(0.70-0.79)$ & $<0.001$ \\
0.58 & 58.57 & 76.02 & 2.44 & 0.55 & 78.2 & 55.6 & $0.74(0.69-0.78)$ & $<0.001$ \\
\hline
\end{tabular}

CEA, carcinoembryonic antigen; LMR, lymphocyte-to-monocyte ratio; AGR, albumin-to-globulin ratio; PLR, positive likelihood ratio; NLR, negative likelihood ratio; PPV, positive predictive value; NPV, negative predictive value; CI, confidence interval; AUC, area under curve. 
Figures

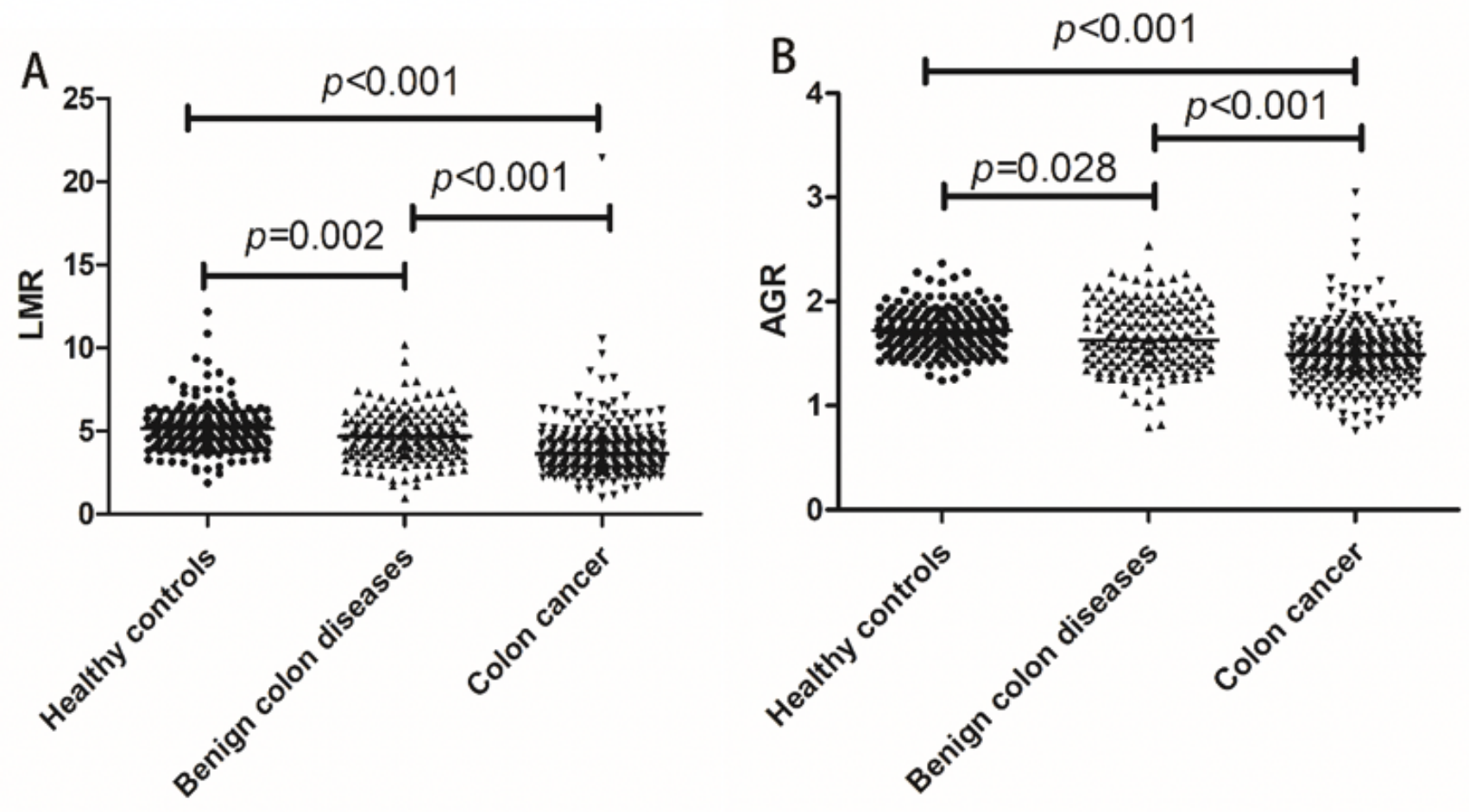

Figure 1

Comparison of LMR and AGR among three groups. A, LMR; B, AGR. 

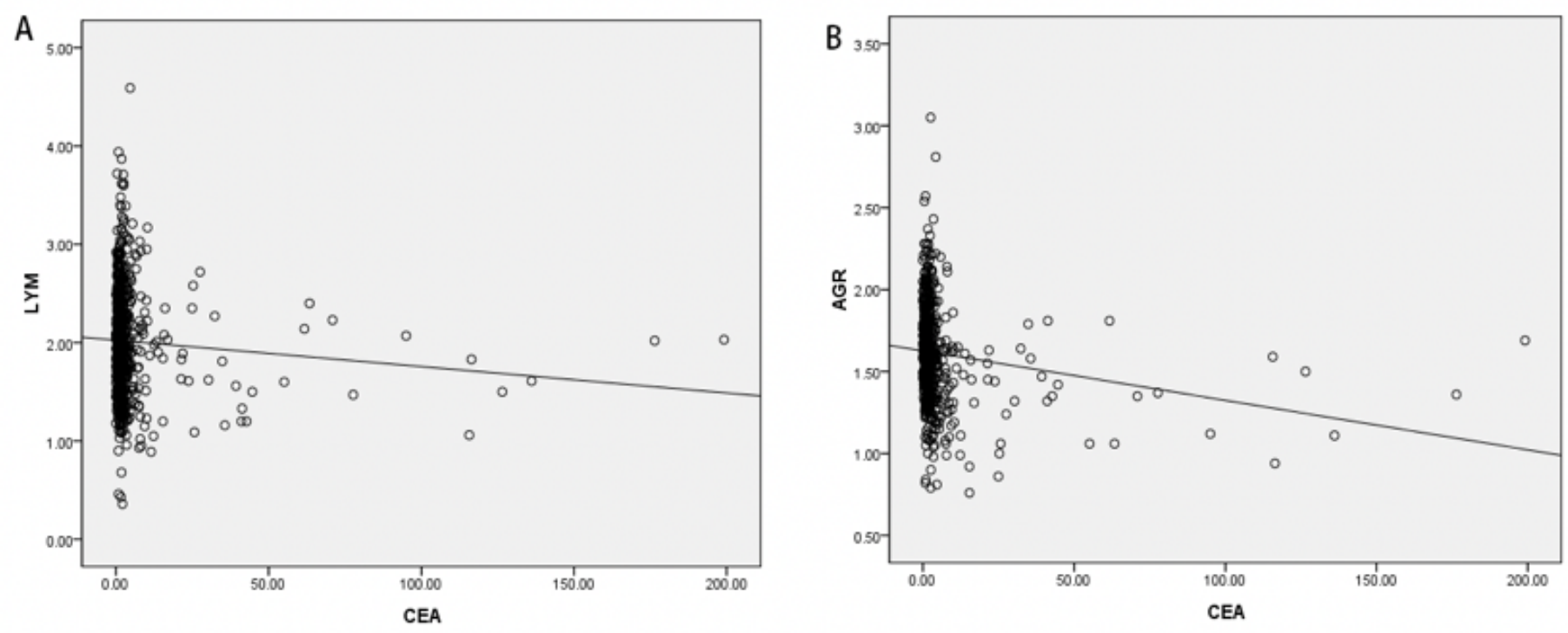

Figure 2

Correlation analysis of LMR, AGR and CEA in patients with colon cancer. A, LMR and CEA in patients with colon cancer; $\mathrm{B}, \mathrm{AGR}$ and $\mathrm{CEA}$ in patients with colon cancer. 


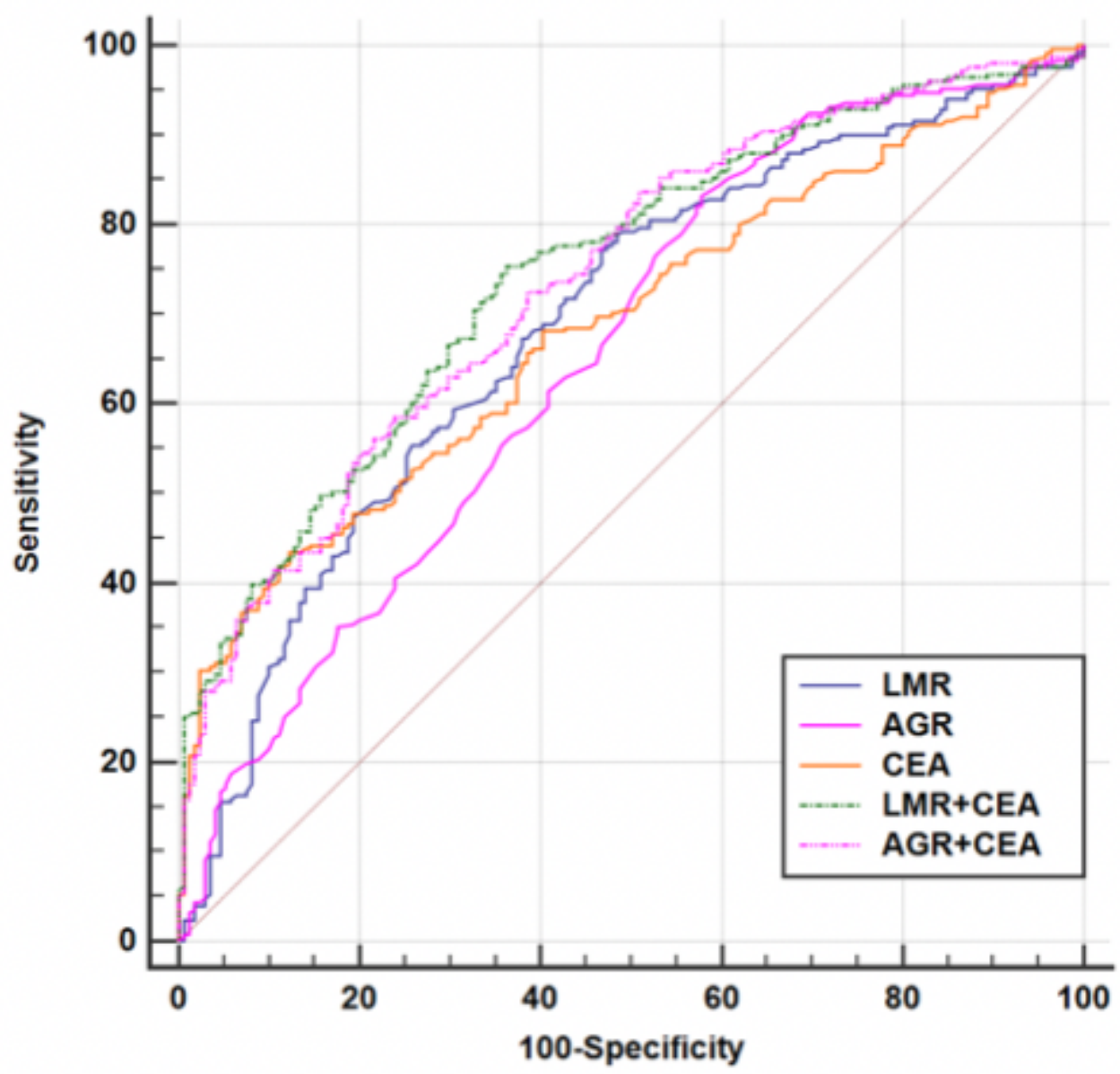

Figure 3

The diagnostic value of LMR, AGR and CEA used alone or in combination for distinction between colon cancer from benign colon diseases. 